\title{
Semantics, Features, and Applications of the Viewpoint Abstraction
}

\author{
Renate Motschnig-Pitrik ${ }^{1}$ and John Mylopoulos ${ }^{2}$ \\ 1 Department of Applied Computer Science and Information Systems \\ Rathausstrasse 19/4 \\ 1010 Vienna, Austria \\ email: motschnig@ifs.univie.ac.at \\ 2 Department of Computer Science, \\ University of Toronto, \\ Toronto Canada M5S 1A4 \\ email: jm@cs.toronto.edu
}

\begin{abstract}
Partitioning information bases such that their contents may be viewed from different situations and represented and processed in different contexts, constitutes a fundamental concern in various disciplines of computer science. Not surprisingly, numerous notations and techniques support certain aspects of the viewpoint abstraction. This paper motivates the use of a well defined terminology and framework regarding basic notions accompanying the viewpoint abstraction, such as contexts, perspectives, situations, and relativism. Furthermore, it establishes the cognitive and linguistic evidence on the usefulness of considering multiple views. A previous paper introduced a generic framework for contexts in order to provide a common kernel for the modelling of information base partitions. This paper demonstrates the embedding of the framework into an extensible, structurally objectoriented data/knowledge model and illustrates the applications of contexts and their accompanying mechanisms for authorization and change propagation to the modelling of (database) views, (software engineering) workspaces, and versions.
\end{abstract}

\section{Introduction}

Classical abstraction mechanisms have proved to be indispensable means for managing the complexity in various notations 1 employed for programming and conceptual modelling. In general, an abstraction is a simplified description, of some subject matter that emphasizes some details or properties while suppressing others [Shaw84]. As a result, the simplified description may be more easily understood and used. Previous research (e.g. [Smith 77], [Brodie84], [Hull87], [Motschnig-Pitrik92, 93] [Storey93]) has concentrated on the classical abstractions like classification, aggregation, and generalization/specialization, that by now are well-accepted semantic modelling concepts. One abstraction that is by far less well understood is that of providing partial information on conceptual entities by viewing them from different viewpoints or situations and representing them in different contexts [Mylopoulos95], [MotschnigPitrik95]. Nevertheless, the notion of viewpoints has been considered in quite a few

In the following, the term notation will be used in a generic sense to subsume terms like language, diagrammatic technique, and data/knowledge model. 
formal notations of computer science stemming from areas such as programming languages, software development, database modelling, and knowledge representation. In these areas, the viewpoint abstraction appears under the guises of workspaces for the support of cooperative work, views delineating data for particular user groups, or knowledge base partitions typically constraining the problem space with respect to reasoning to a manageable size. Most proposals provided in the literature and in software products, however, deal with only some of the issues of a comprehensive viewpoint mechanism. Furthermore, they use disparate terminology, such that their features can hardly be compared. Yet, as will be argued shortly, the viewpoint abstraction, like the other classical abstractions, has a common, uniform kernel whose support proves beneficial for a number of well defined tasks, such as the support of database views, sof tware engineering workspaces, AI topics, versions, etc. .

We see information bases as a generalization of terms such as (object-oriented, deductive, active, multimedia, or just traditional) databases, knowledge bases, hypertext bases, etc. . The construction of such information bases assumes an information model which, again, is seen as a generalization of the the terms data/knowledge model, conceptual model, or knowledge representation scheme. Proper use and management of an information base is supported by an information base management system which offers an information model along with efficient query and transaction processing facilities. The exact nature of these facilities may well be influenced by structuring mechanisms such as the one proposed here.

The purpose of this paper is to embed a formal framework for the viewpoint abstraction (for more details see [Mylopoulos95], [Motschnig-Pitrik95]) into an object-oriented notation and to illustrate in what ways the framework provides a unifying notion and hence support of well known applications such as views, workspaces and versions. In addition, the paper

* motivates the consideration of viewpoints as a useful abstraction that facilitates the construction and maintenance of large information bases and leads to models that more transparently model (some aspects of) the real world. This issue encompasses the comparison of viewing with related notions;

* explores results from cognitive psychology and linguistics that point to the benefits of organizing information along contexts such that each context addresses a particular situation in the real world;

* shows some benefits of integrating the generic framework for contexts [Mylopoulos95] into a particular information model, for example an objectoriented one;

compares our framework with related work and demonstrate how our model addresses and provides more uniform support of several features of applications of the viewpoint abstraction, in particular views, workspaces, and versions.

The paper is organized as follows: the next section introduces the viewpoint abstraction and explores its cognitive and linguistic grounding. The generic context framework ([Mylopoulos95]) is reviewed in section 3 and illustrated by a simple example. Section 4 shows the embedding of the generic framework into an extensible, structurally object- 
oriented language. While we use Telos, the principles and insights gained from the embedding apply to most object-oriented notations. Nevertheless, as will be shown shortly, the extensibility of Telos allows for a particularly straight-forward incorporation. Applications of the context framework, such as its use for the modelling of (database) views, (software engineering) workspaces, and major aspects of versions, are discussed in section 5. This section further compares our approach with related work. Finally, the conclusion rounds up the paper and points to issues for further research.

\section{$2 \quad$ Terminology and Grounding}

\subsection{Terminology}

In the literature, several related terms, such as perspective, view, context, vista, space, world, role, and topic are employed to capture two accompanying notions used in the context of information base partitions. In [Motschnig-Pitrik95] the terms context and perspective are introduced and it is shown how these notions relate to the application dependent terms employed in individual approaches to view modelling. In this paper we give a more detailed scenario illustrating the appliability of contexts and perspectives in an object-oriented environment. As a starting point, consider the meaning of perspectives as derived from Webster's Dictionary, where a perspective is described to mean: an "aspect of an object of thought from a particular standpoint". Based on this definition and on the insights gained from the meaning of synonyms and related terms listed above, let us adopt the following as a working definition on perspectives:

A perspective is a symbolic structure encoding information about a conceptual entity as viewed from a particular standpoint. Borrowing from [Levesque84], a standpoint, more technically, will be referred to as a situation. Due to the emphasis on a particular situation, the information encoded in a perspective is considered partial when compared with the representation of the conceptual entity regardless of that situation. Typically, multiple perspectives on the same conceptual entity coexist in one model and thereby provide alternative descriptions of that conceptual entity. This multiplicity can be ascribed to the fact that the contents of perspectives are determined by viewing a conceptual entity from particular situations. Moreover, not only the contents but also the most appropriate representation of a perspective may be determined by a particular situation. Thus the reason for specifying a perspective is basically extrinsic, determining the inclusion of a relevant subset of the characteristics of the entity. Note that he term conceptual entity is used synonymously with the 'object of thought' taken from the quote in Webster's dictionary and is intended to mean any semantically meaningful entity. The relationship between a perspective and the corresponding conceptual entity is referred to as perspective-on and its inverse is called has-perspective.

In a specific case the conceptual entity itself, without (seemingly, for the moment) any regard to a specific situation may be represented by a symbolic structure within a model. In that case the symbolic structure will be referred to as the primary perspective, in order to allow to distinguish it from the others. In this respect, in the above definition the term partial should be understood in contrast to (approachably) complete information as typically included in the primary perspective or, in the case the latter is not represented, as given by the union of all perspectives on some entity. In fact, multiple intentions behind expressing partial information can be identified, such as setting the focus by 
ignoring issues not relevant to a specific situation, modelling limited availability of or restricted access to certain kinds of information, describing situations with not yet established further evidence as in reasoning processes, or viewing an entity from just one instant of time as opposed to tracking it throughout its entire life cycle.

Complementary to mapping a conceptual entity into perspectives according to situations, it often appears useful to represent the situations themselves within the model. This is specifically the case if some subject matter consisting of several conceptual entities is to be viewed from the same situation. The latter then can be interpreted as a frame (space), enclosing the conceptual entities viewed. In accordance with [Schneider80], we refer to the denotation of a situation in a model as a context. Thus, a context typically is a meaningful slice of the model or, in a special case, represents the universal context. In fact, our usage of the term perspective corresponds to what Schneider called a view. The latter term, however, we tried to avoid since it is heavily overloaded in current approaches.

Note, that decomposing information bases with contexts is orthogonal to the classical notion of the decomposition of a system into subsystems which is driven by the goal of producing a good system's decomposition (see for example [Yordon79], [Wand91]). Indeed, contexts and subsystems differ in so far as a subsystem is it intended to provide a unique and complete specification of some part of the model, covering, at its best, the representational need of all situations. On the contrary, a context, in general, is constructed with different intents in mind, such as to provide a customized view of the system for a particular user's group, to delineate the information necessary for accomplishing a particular task, or to focus attention on a particular topic and abstract from other information.

\subsection{The Cognitive Evidence for a Viewpoint Abstraction}

Besides computer science, architecture, psychology and, in particular linguistics have acknowledged the importance of the viewpoint abstraction. Consider, for example, the practical utility of the traditional notion of perspective as applied by architects in the design of artifacts. In order to provide the complete picture of, say, a building, several perspectives have to be provided.

Results from cognitive psychology indicate improvements in the mental performance of humans when being confronted with information organized along situations or viewpoints. We believe that such results provide useful guidelines on the choice of semantic concepts in those situations in which understandability by humans is an issue, such as in conceptual modelling. Therefore we briefly state some findings from Cognitive Psychology [Anderson90] that offer hints on the ways perspectives and contexts support and improve human information processing.

Consider, for example, an experiment designed to test the effects of the provision of foci of attention [Rothkopf66]. In a text retrieval task, Rothkopf had three groups of subjects read a long text, each under different conditions: while the text of the first two groups contained questions interspersed every few pages and relevant to the pages following or, respectively, preceding the questions, the third group read the text without any such questions. Furthermore, the first group was advised to answer the questions after reading 
the pages and the second to read the text with the questions in mind. In a final test on the whole text the third group answered correctly $30 \%$ of the test items, whereas the groups whose questions previewed or reviewed the text answered $72 \%$ of the test items relevant to their questions and $29 \%$ or $42 \%$ of the irrelevant items, respectively. This indicates that considering specific standpoints or situations proves beneficial for understanding larger amounts of information.

In general, many of the effects, such as the one above, in which a more constrained situation can be mastered better than a more convoluted one can be explained by the fact that the processing of new information requires attention. The latter is defined in [Anderson90] as being a "very limited mental resource that can be allocated to at most few cognitive processes at a time." Consequently, all means of focusing attention to some specific situation--imagine to listening to only one of two people talking at once or to concentrating on a single task at a time--will improve performance in that situation. Besides being important on such a broad basis as focusing attention, the merits of constraining information to a set of facts relevant to a specific situation can equally well be ascertained at a more syntactic level. Subjects who were presented sentences on famous people could respond faster to questions about these people than subjects who, in addition to the same sentences, were also presented fancy facts about the same famous people [Lewis76]. Experiments, like the one described, directly point to the fact that information not being relevant in a situation is not simply redundant but even has deteriorating effects on the processing of the relevant fraction.

\subsection{The Linguistic Evidence for a Viewpoint Abstraction}

In linguistics, the notion of a context is a central one, since the context determines the meaning of expressions. For example, P. Gerstl [Gerst193, p.82,83] writes: "The context in which an expression occurs determines its meaning. The context at a specific point of time during a discourse consists in a typically large number of factors that reflect dependensies between knowledge elements. Formally speaking, factors are partial relations that connect intermediate results of the interpretation process. ... Since factors themselves are derived from knowledge elements, they differ in the way they are represented in the system. Some are explicitely 'coded' as attributes of lexical units or concepts, others result from the application of rules. ... Besides linguistic factors due to global syntactic requirements, (...), some derive from background knowledge, others from the discourse situation or from conventions governing successful communication." This quote nicely demonstrates the analogy between the linguistic and computer science notion of relativity: the linguist's 'interpretation of expressions within a context' corresponds to the computer scientist's ' transaction execution with respect to a context'.

\section{Review of an Abstract Context Model}

In [Mylopoulos95] a generic framework for partitioning information bases into possibly overlapping contexts has been proposed and will briefly be reviewed in this section. The proposal makes minimal assumptions on the underlying information base model, notably that an information base consists of named, interconnected information units, operated upon by transactions that are triggered by users. The main contribution of the context model rests with the features chosen for the context mechanism, namely: 
* relativism, in the sense that the same conceptual entity may have different perspectives as well as names in different contexts;

* relativized transaction execution, meaning that transactions are executed with respect to a context;

* authorization, whereby every context is associated with an authorization predicate such that both content-dependent as well as content-independent authorization is provided;

* change propagation, allowing one to define propagation channels between context and optionally to constrain these channels to transmitting changes that depend on the user and the transaction that issued the change.

\subsection{Definitions and Basic Postulates}

In the terminology of the abstract context model, an information unit (hereafter just unit) is the basic building block for information bases. A unit might refer to an entity, object, attribute, relationship, agent, rule, method, record, text file etc. Let Units be the set of all possible units. Likewise, we define Predicates to be a set of Boolean expressions, possibly with free variables, used as components of context definitions, while Users is the set of potential users who may access or operate on an information base, Transactions is the set of all possible atomic operations on contexts, and propagationLinks are all potential interconnections between contexts along which changes may be propagated.

Contexts are special units representing a decomposition of the information base. The definition of each context includes four components which define respectively the

- contents of the context,

- the local names (lexicon) used for units in the context's contents,

- authorization rules based on combinations of different users and transactions, and

- propagation links specifying contexts which receive changes from or propagate changes to the context under definition :

P(Units) $\times($ Identifiers $\rightarrow$ Units $) \times$ Predicates $\times$
$\times$ PropagationLinks $\supseteq$ Contexts

The selector function contents takes as argument a context and returns the set of units included in that context

contents: Contexts $\rightarrow \mathrm{P}$ (Units) ${ }^{2}$

When a unit $\mathrm{o}$ is an element of the contents of context $\mathrm{c}$, we will say that $\mathrm{o}$ is visible in c. Note that units provide both an IB-wide unique identification of their referent conceptual entities as well as a context dependent representation of their referents, $i$. e. perspectives. In order to provide these two functions, each unit is constructed to consist of a locally unique identifier and a context dependent reference to a perspective. In some

${ }^{2} \mathrm{P}(\mathrm{Set})$ stands for the powerset of Set. 
more detail, a unit's global identifier (or a local identifier, tagged by the context name) uniquely determines the unit's referent such that within one IB no two conceptual entities are referred to by the same unit. A unit's reference to a perspective, however, is context dependent. Thus, considering the example in figure 1 , ' unit 3 ' denotes the conceptual entity of a particular person called chryss which may be modelled by different perspectives in the contexts $c 1$ and $c 2$, respectively.

Identifiers are special units consisting of character sequences used as external names for other units with respect to a given context. The selector function lexicon maps each context to its lexicon of identifiers and their referents with respect to that context

\section{lexicon: Contexts $\rightarrow$ (Identifiers $\rightarrow$ Units)}

Thus, (lexicon(c)) ('john') evaluates to the referent of the external name ' john' with respect to context $c$. This referent identifies the conceptual entity called ' john' with respect to context $\mathbf{C}$ and points to the respective perspective. In the sequel, we will use simply john when the context is obvious, as a shorthand for the above expression. Note that the same units may have different identifiers (i.e. external names) with respect to different contexts and, conversely, the same external name may refer to different units in different contexts. Renaming of units, though, should be done with great care since it hides the externally visible semantic ties between perspectives.

Likewise, authorP is a selector function which selects for each context $\mathrm{c}$ and usertransaction pair $(u, t)$ a predicate which determines whether user $u$ is authorized to execute transaction $t$ within context $c$ :

\section{authorP: Contexts $\rightarrow$ (Users $\times$ Transactions $\rightarrow$ Predicates)}

In a similar vein, propagationfrom and propagationTo are selector functions which associate each pair of communicating contexts with an authorization predicate:

$$
\begin{array}{r}
\text { propagationfrom: Contexts } \rightarrow \text { (Contexts } \times \\
((\text { Users } \times \text { Transactions) } \rightarrow \text { Predicates)) } \\
\text { propagationTo: Contexts } \rightarrow \text { (Contexts } \times \\
\text { ((Users } \times \text { Transactions) } \rightarrow \text { Predicates)) }
\end{array}
$$

propagationfrom indicates a communicating context that is supposed to propagate update operations to the context under definition. In analogy, propagationTo indicates which context shall be sent changes from the context under definition. In both cases, propagation proceeds selectively in so far as the changes to be sent or received may be filtered by an authorization predicate such that only specific changes are transmitted/accepted. As a result, a change operation in one context effects another context only if both communicating context agree on the propagation.

Figure 1 illustrates the above definitions with a simple example which contains two contexts $c 1$ and $c 2$, each including three units, along with their respective lexicons, authorization predicates and change propagation links. 


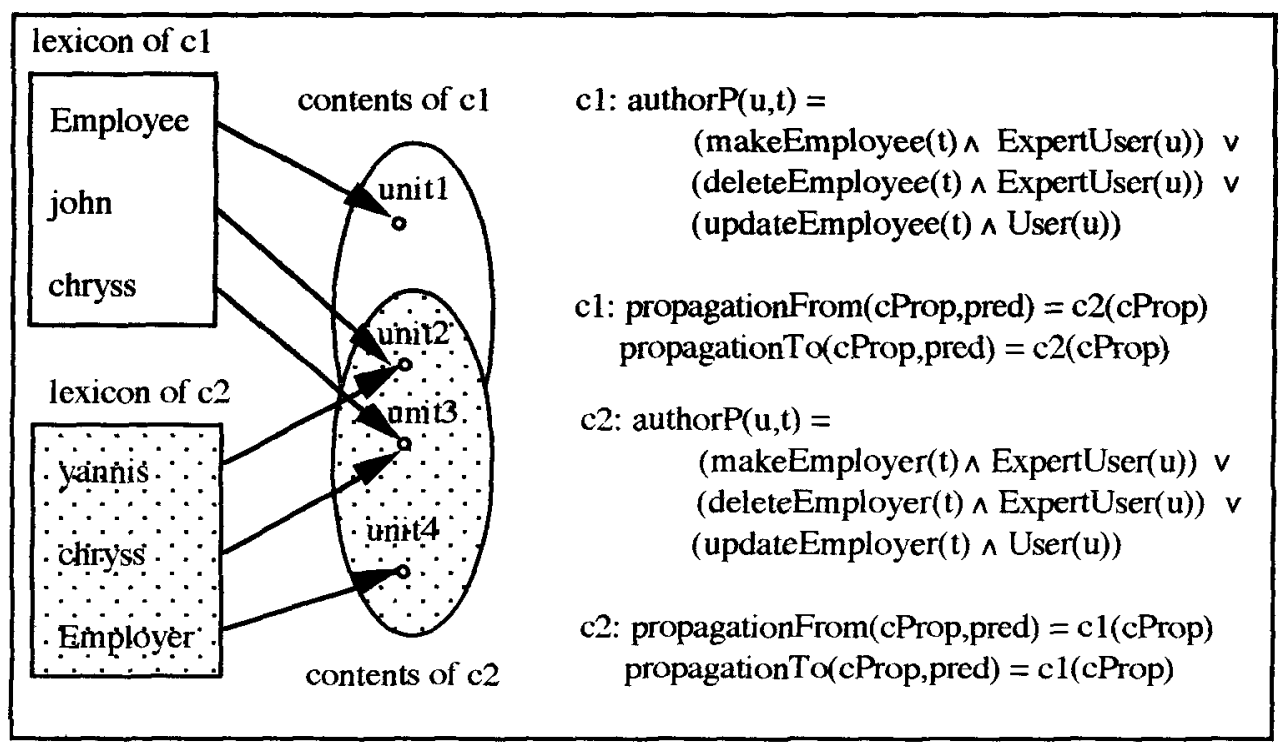

Fig. 1: Example showing the features of the context model

The contents of the context $\mathrm{c} 1$, for example is defined as:

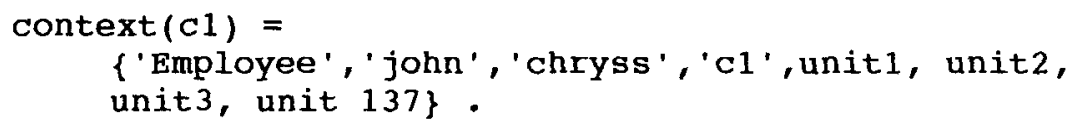

The lexicon for $\mathrm{cl}$ has the following name-unit identifier pairs:

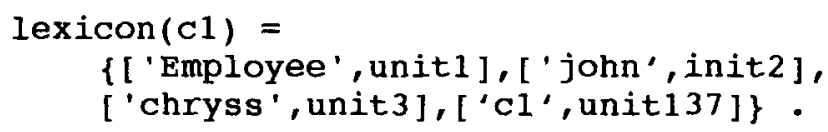

According to the figure, 'chryss' is used synonymously in the two contexts and so are the identifiers 'john' with respect to context $\mathrm{cl}$ and 'yannis' with respect to context $\mathrm{c} 2$. The authorization predicate of $\mathrm{c} 1$ authorizes users having the role ExpertUser to execute makeEmployee and deleteEmployee transactions, All users having the role User (or a role that implies the user role, such as ExpertUser) may execute updateEmployee transactions. A similar predicate is accociated with the context $c 2$. Further, propagation links are specified as to allow for unconstrained bidirectional propagation of changes.

The special context null represents the empty context. Note that an empty context may still contain units because it includes built-in units that need not be created explicitly by users of an information base. Moreover, the only transactions that can be executed with respect to the null context are Query transactions (which retrieve information from, but don't change the contents of nul1). This means that the contents of the null context can't be changed:

$$
\operatorname{authorP}(\operatorname{null})(u, t)=\operatorname{Query}(t)
$$


An information base is a collection of contexts

InformationBases $=\mathrm{P}$ (Contexts)

\subsection{Operations and Transactions}

\subsubsection{Primitive Operations on Contexts and Information Bases}

Operation on Information Bases. Information bases can be treated as special contexts in the sense that their contents may only contain contexts. Like other contexts, information bases include an authorization and change propagation component. New IB's are created through the operation

$$
\text { newIB( ) }
$$

which creates and returns a new IB that contains the single context null which contains all the built-in units for the new IB. Note that newIB() is the only operation which is not executed with respect to a context. Thus, an information base is a collection of contexts

$$
\text { InformationBases }=\mathrm{P} \text { (Contexts) }
$$

Following some object-oriented notations, we also postulate that each context is an element of its own contents and can be referenced through the special local identifier self:

$$
(\forall c / \text { Contexts })[c \in \text { contents }(c) \wedge \text { self wrt } c=c]
$$

Operations on Contexts. There are two sets of primitive operations on contexts. Firstly, there are operations which create new contexts from sets of units or in terms of existing contexts. Secondly, there are operations for modifying the contents, lexicon and authorization predicate of a context.

newContext (unitSet, unitPairSet,pred(u,t), $\operatorname{chProp}(\mathbf{c}, \operatorname{pred}(\mathbf{u}, t))$ creates a new context containing all elements of unitset, having lexicon unitPairSet, authorization predicate pred, and change propagation chProp. The new context is added to the contents of the context wrt which the current transaction is executing:

$$
\begin{array}{r}
\text { newContext: } P(\text { Units }) \times \text { P(Identifiers } \times \text { Units) } \times \\
\times \text { Predicates } \times \text { Propagationtinks } \rightarrow \text { contexts }
\end{array}
$$

clone(c) creates and returns a new context which "clones" the contents and lexicon of $c$, while its authorization predicate is set to true and no propagation links are defined.

Context addition $(\oplus) . c \oplus c^{\prime}$ creates and returns a new context whose contents are defined as the set-theoretic union of those of $c, c^{\prime}$. The definition of context addition [Mylopoulos95] implies that when an identifier has different referents in c, $c^{\prime}$, its referent with respect to $\mathrm{c}$ takes precedence within $\mathrm{c} \oplus \mathrm{c}^{\prime}$. 
Likewise, the operations context product and context difference are specified as adaptations of their set-theoretic operation cousins.

The primitive operations provided for changing a context component include ones for inserting/removing entries from the contents and lexicon of a context.

\author{
insertContents(c,unitSet) \\ removeContents(c,unitSet) \\ insertLexicon(c,tupleSet) \\ removeLexicon(c,tupleSet)
}

In addition, operations are provided for assigning to and removing from a context an authorization predicate, for adding/removing a propagation link, for changing incrementally the authorization predicate and/or propagation channels, etc. Furthermore, our proposal assumes that an information base comes with two sets of operations:

* Primitive operations for different classes of units, including operations on contexts described above;

* Transactions on the information base.

\title{
3.2.2 Primitive Operations on Different Categories of Units
}

Primitive operations define what operations make sense for a particular unit in a particular context. Such operations are are defined along with each unit class. Note that a unit may belong to different classes in different contexts, and therefore admit different sets of primitive operations. For instance, a unit may be a text file in one context and a $\mathrm{C}++$ object in another. This means that the same unit may have different associated representations in different contexts but there is an information base-wide mechanism for keeping track of corresponding representations of the same unit. One such mechanism may be to associate to each representation of a unit in a context an internal, global identifier which uniquely identifies the unit being represented. A second alternative would be to maintain tables of correspondences between the (local) internal identifiers of units in different contexts.

\subsubsection{Transactions}

Transactions define meaningful operations for the whole information base. They are defined in terms of primitive operations on units including contexts and compositional constructs such as sequencing, conditional, iteration. Transactions are always executed with respect to (hereafter wrt) a particular context. and can access and/or effect change only to that context.The context with respect to which a transaction execution is carried out is referred to as its execution context. An execution context provides, through its lexicon, the symbol table used by a transaction execution. As with other modern database transaction models, our transaction model is nested and assumes rollback if a transaction execution fails.

Transactions can be thought as classes (in the sense of object-oriented information models) and can therefore be organized in terms of a generalization hierarchy. We shall assume that this organization is based at least partly on the generalization hierarchy of their parameter lists [Mylopoulos80]. For instance, the Assignsal (emp: Employee) transaction might have specializations depending on 
whether its argument is a secretary, Manager, Designer or Receptionist or one of the specializations of these Employee classes.

We may assume that there are built-in transactions which support basic query, insertion, deletion or update operations. These may be refined to form specialized query, insertion, deletion or update operations; alternatively, these may be combined to form composite transactions which carry out combinations of the primitive operations. The left part of figure 2 shows an example of such a hierarchy for the use with the Telos context framework presented in the next section. Note that the arrows in the figure point in the direction of authorization implication. Names in capitals denote operations that are predefined in Telos, while the names in small letters distinguish user defined transactions. In order to determine whether a given transaction is authorized to be executed in a given context, this context's authorization predicate is examined. The transaction is executed in the case that it is authorized explicitly by an authorization predicate, or its authorization can be derived by following the authorization implication links of the transaction lattice.

In the same spirit, we assume that users of the information base are instances of user classes (or user roles), such as SystemAdministrator, Manager, ExpertUser, User, etc. which are also organized in an authorization implication lattice.
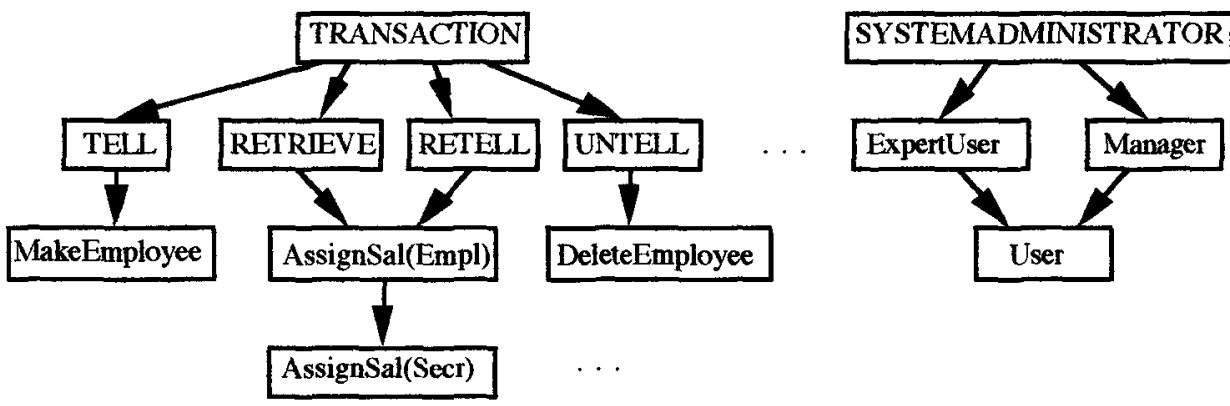

Fig. 2: Example of a transaction lattice (left) and a user role lattice (right) for authorization implication.

\section{An Information Model with Contexts}

In order to illustrate how existing object-oriented information models can be extended to support contexts, this section shows the embedding of contexts into the Telos framework. We chose Telos mainly due to its extensibility of language constructs (explained shortly), object-oriented nature, associated assertion language capable of expressing, for example, authorization predicates, and its transparent high-level notation having a precise, logically grounded low-level counterpart.

Telos is a knowledge representation language originally designed for information system development applications [Koubarakis89, Mylopoulos90, Jarke92]. Its representational framework is a generalization of graph-theoretic data structures used in semantic 
networks, semantic data models and object-oriented representations, supporting classification, generalization and attribution. The assertional component of the language includes linguistic facilities based on First Order Logic for declaring constraints and deductive rules. Its novel features include the treatment of attributes as first-class citizens, the extensibility provided through metaclasses and a powerful classification mechanism [Motschnig-Pitrik92] and the special representational and inferential facilities for temporal knowledge (which will not be dealt with in this paper). We'll introduce Telos incrementally, pointing out extensions required in order to support the context mechanism proposed here. The main objective in adopting the context mechanism has been to use as much of the basic Telos framework as possible, in order to minimize extensions to the language.

\subsection{The Family Metaphor}

A Telos information base consists of propositions, which are either individuals (entities) or attributes (named, binary relationships, represented by triples [ source, label, destination ${ }^{3}$ (or [from, 1 abel, to]). Assertions are treated as special individuals, while instanceof (in) and is A relationships are treated as special attributes. For purposes of introducing a context mechanism into the language, Telos information bases can be viewed at two levels of granularity, the proposition level and a corresponding family level. At the proposition level, a Telos information base is simply a collection of propositions. Moreover, everything is a proposition. Changes to the state of the information base simply amount to insertions/removals of propositions.

At the family level, on the other hand, an information base is viewed as consisting of several overlapping families of (interrelated) propositions each defined by one parent proposition, along with all attributes that have this proposition as source, all instanceof (in) links connecting these attributes to classes and all components of attributes in the family, including instantiated classes. The contents of a family provides information on the relationship of the parent proposition to other propositions, along with instantiation information that offers possible interpretations of parents and their associated relationships. A family is intended to be a minimal meaningful collection of propositions. Families are to be seen as the molecules out of which one constructs an information base. As an example, figure 3 shows the family having the parent proposition chryss. Note that attributes, such as [chryss, SI\#, 06061507], are treated as potential family parents too, whose components include instance links and attributes and attribute components representing relationships to other propositions (e.g., the attribute [Chryss, SI\#, 06061507] is instance-of [Person, SI\#, Integer ]). If Telos information bases are defined at the family level, operations effecting changes may include insertions, removals of families (each involving the insertion/removal of several propositions) as well as family updates (i.e., changing the set of attributes associated with a particular family).

3 Actually, propositions have a fourth component that defines the lifetime of the entity or relationship represented by the the proposition. As indicated earlier, for reasons of expediency we will ignore the temporal component of Telos. 


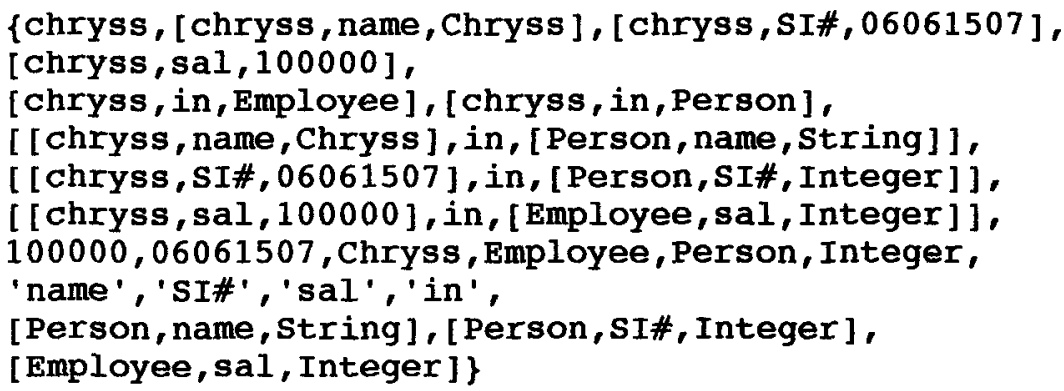

Fig. 3: Example of a family with the parent proposition chryss.

For the rest of the discussion we focus on the family level, since it leads to a more interesting, and powerful, notion of Telos contexts. This is because sets of (related) families can be encapsulated into yet larger building blocks, referred to as neighborhoods and districts. In order to make the notion of a family and a district more precise, we introduce the function family (prop) as a special case of the function district (propset), defined in terms of the auxiliary functions nucleus, comp and closure.district (propset) specifies the set of all propositions constituting a Telos family with parents the elements of propset:

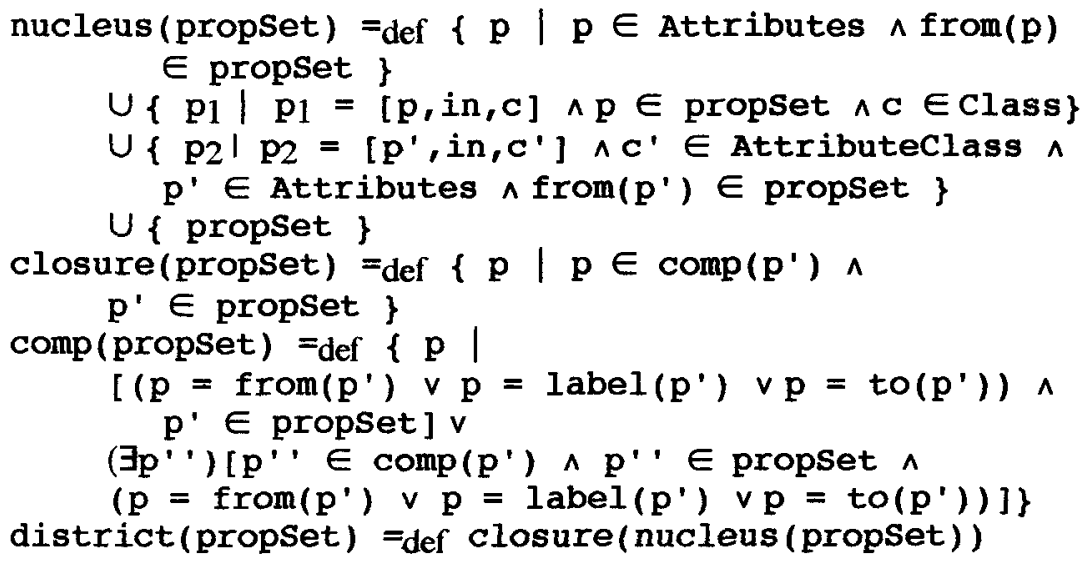

According to this definition, the topology of a district is defined in terms of a set of parents, one or more classes each parent (respectively) is an instance of, and the attributes associated with the parent and these classes, as illustrated above. The definition of a family is derived from that of a district by having propset contain a single proposition only. Thus, districts derived from a single parent proposition are called families.

Figure 4 shows several examples of family creation operations (defined through TELL), all having a single parent, namely the name of the class/token under definition. In particular, PersonClass is defined as a metaclass (whose instances are classes) and a specialization of the metaclass SimpleClass. In addition, PersonClass has an associated attribute class labelled avgAge, declared to be a single-valued, simple class (i.e., its instances are tokens, representing particular binary relationships in the application 
domain). Person, on the other hand, is declared to be a simple class with a name attribute class, a single-valued SI\# attribute class and a avgAge attribute having the value 30. Employee and Employer are defined as specializations of Person (also instances of PersonClass) with additional attributes. Finally, chryss and john are defined as tokens, instances of Employee and Employee as well as Employer respectively, having particular attribute values for attribute classes associated with the class they are classified under. Note that the family being created in terms of TELL TOKEN chryss operation, for example, has been given in figure 3 above.

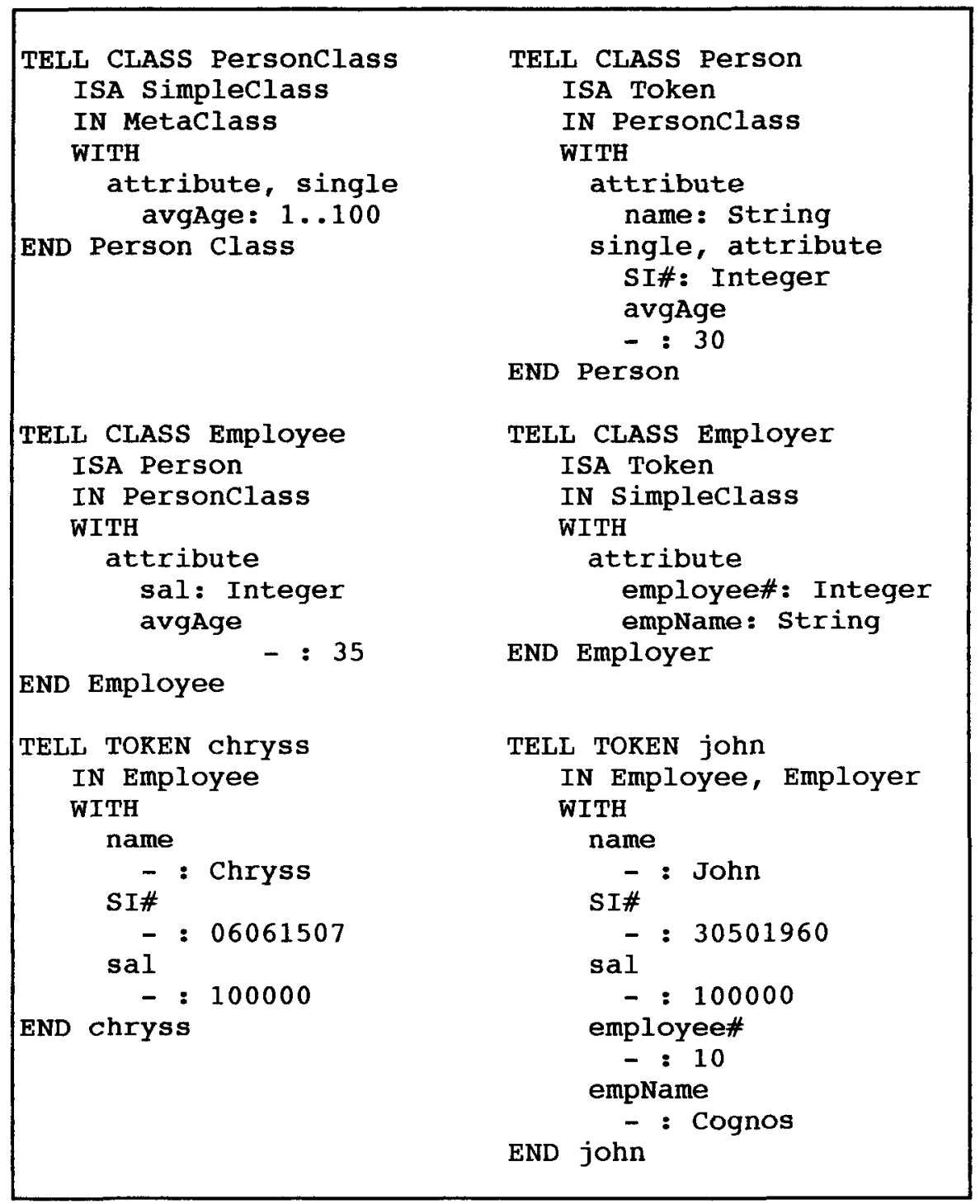

Fig. 4: Telos family definitions 
A final point about Telos, before returning to contexts. Attribute and Single are attribute metaclasses which are used to bestow semantics to attribute classes. Single, for example, can be defined as shown in figure 5. This definition declares the source, label, and destination components of single and associates with it a constraint that states, in effect, that its instances are single-valued attribute classes. This class is instantiated in the definition of the attribute class SI\# to declare that it is single-valued.

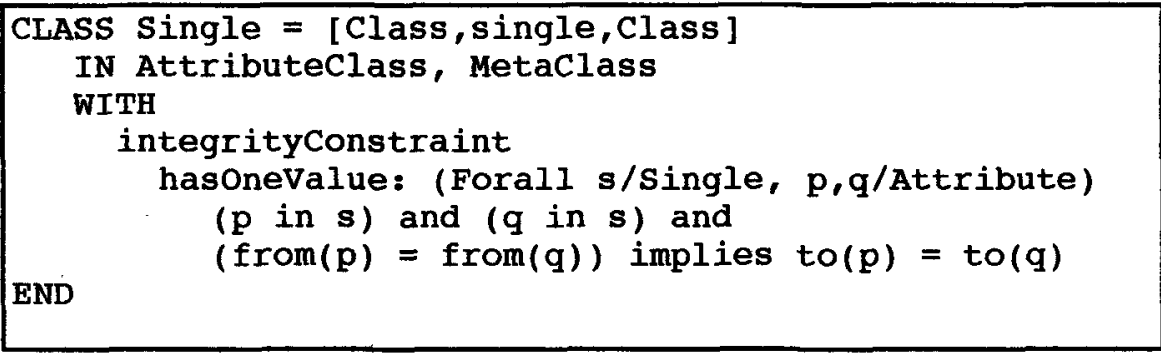

Fig. 5: Defining the attribute metaclass Single

\subsection{Contexts as Family Homes}

In order to introduce contexts in Telos, we begin by defining the class Context, which has all contexts constituting an information base as instances.

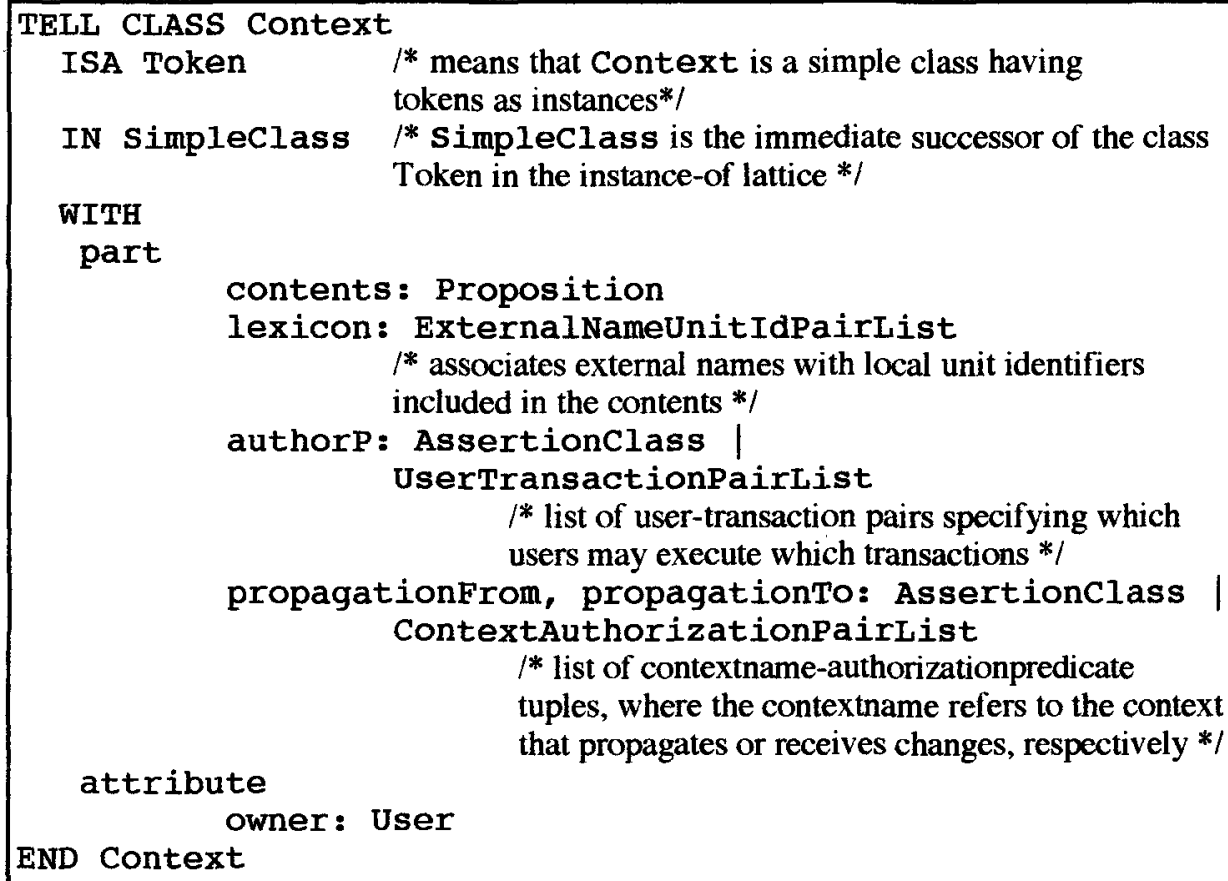

IN Simpleclass $/ *$ Simpleclass is the immediate successor of the class Token in the instance-of lattice */

WITH

$$
\text { part }
$$

contents: Proposition

lexicon: ExternalNameUnitIdPairList

/* associates external names with local unit identifiers included in the contents *I

authorP: AssertionClass

UserTransactionPairList

$1^{*}$ list of user-transaction pairs specifying which users may execute which transactions */

propagationfrom, propagationTo: Assertionclass |

ContextAuthorizationPairlist

* list of contextname-authorizationpredicate tuples, where the contextname refers to the context

attribute that propagates or receives changes, respectively */

END Context

owner: User

Fig. 6: Definition of the class Context 
In addition, we assume that Context, like other built-in Telos classes are all in the empty context, null, and are therefore present in all other contexts too.

The second extension to Telos exploits the obvious correspondence between contexts and families, based on the observation that both define collections of propositions. However, a context has associated supporting structure (contents, lexicon) and offers mechanisms for encapsulation, authorization and change propagation. Families, on the other hand, are mere collections of propositions that constitute an information base neighborhood. It therefore makes sense to treat a family as a context, thereby endowing it with contextrelated structure and mechanisms.

As an example demonstrating the definition of a context based on a particular family, namely family (Person) consider the creation of the token personcontext as an instance of the class Context. Assume that personcontext is created within an enclosing context called enterprise as shown in figure 7.

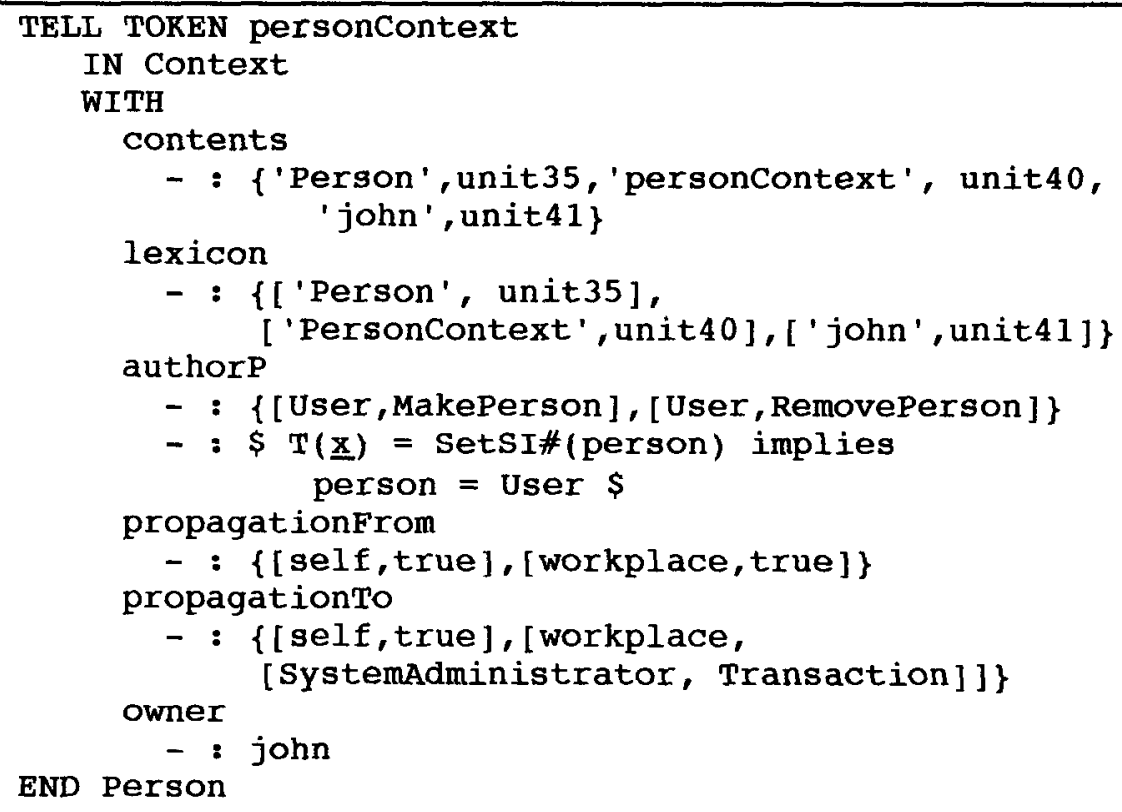

Fig. 7: Defining Person as a context.

We assume that Person is being created within the context enterprise, so self refers to enterprise. Also, we will use this to refer to the newly created context. Note that the contents and the lexicon for family contexts are created automatically. Further, the redefinition declares authorization such that all users having the user-role User may execute the MakePerson and the RemovePerson transactions (regardless of the particular parameterlists). The transaction SetSI\# (person), however, may be executed only by users operating on their own records. In addition, the definition declares propagation channels to and from enterprise as well as workplace. 
Furthermore, changes affected by transaction executions initiated by users that carry the role of systemadministrator are specified to be propagated from the Person context to workplace.

A TELI operation then creates a new family and (optionally) declares it to be a context. The family itself may be visible and may be changed in many other contexts. It is often useful to use the original family context as homebase (home) for the family, which accumulates all changes effected elsewhere thereby keeping track of the family status. In general, we envision each family having zero or more homes which are characterized by the fact that they receive change propagations effected elsewhere.

One of the advantages of assigning a newly created family to a home is that the constraints and deductive rules associated with that family may then remain private to the family home and need only be checked when the home is first created or is subsequently updated. This situation is in sharp contrast with that found in the current definition of Telos, where constraints and deductive rules are global to an information base and are therefore checked any time there is an update anywhere in the information base. 4

\subsection{Contexts Beyond the Family Home: Neighborhoods and Districts}

We now turn our attention to other useful collections that might be encapsulated in terms of contexts. The definition of Telos includes functions which make it possible to fetch all instances of a class $c$, in terms of the expression $c$. instances, all classes of which a proposition $\mathrm{p}$ is an instance, $\mathrm{p}$. in, all specializations of a class $\mathrm{c}, \mathrm{c}$. spec, and all generalizations of a class $c, c$.isA. The result of using any combination of these four functions as multiple parent propositions in order to derive a district is referred to as neighborhood. Neighborhoods being constructed such that the parents are, for example, a classname and its chain of super and/or metaclasses, are particularly useful in the construction of contexts since they encapsulate intimately related fragments of an IB. So are neighborhoods that contain as parents a classname along with subclasses and/or instances of the respective class.

In general, Telos contexts (containing more than one family) can be constructed in one of three ways: by a context operation (compare section 4), by explicitly enumerating the families or their parent propositions to be included, or by a query expressed in the Telos query language ([Koubarakis89]). In all three cases the contents of a context is specified as the value of its contents attribute. As an example of a context created by the context addition operation with regard to the two family contexts Employee and Employer, consider the context workplace given in figure 8 and assume it is created within the context enterprise. The contents of workplace is specified by context addition and hence includes the contents of its two parent contexts. In addition, workplace makes use of generalization hierarchies of user roles by authorizing expert users, Expertuser, (assumed to be a subclass of User) to call the transactions MakeEmployee, RemoveEmployee, MakeEmployer, RemoveEmployer. Any user may call updateEmployer and UpdateEmployee. Furthermore, workplace declares a bidirectional propagation channel from/to enterprise and

4 In short, homelessness is computationally (...as well as socially!) undesirable. 
from/to Employee and Employer. Changes propagated from the Test context are specified as not to be considered within the workspace context.

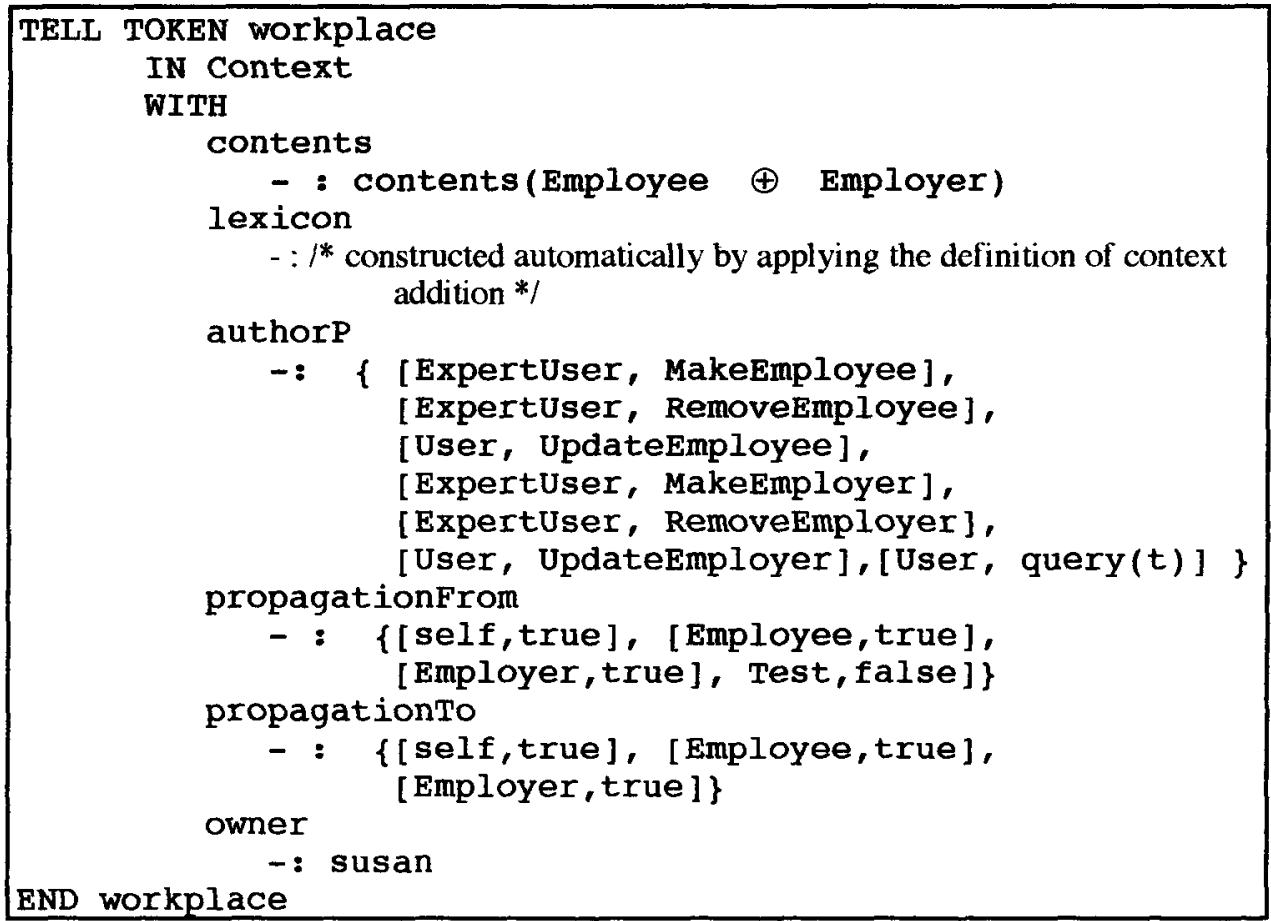

Fig. 8: Example of a context containing multiple families

This example demonstrated in which way the home metaphor can be extended to other useful categories of contexts. By analogy to home contexts, we defined an example of a district context where changes to a family's state are propagated back home. Carrying this idea further, one could define town contexts which are global collections of several homes, neighborhoods, and districts, having a common function within an information base.

\section{Applications and Related Work}

The context model presented above is intended to provide a unifying, generic framework for applications which rely on partitionings of an information base, such as (software engineering) workspaces, (database) views, (knowledge base) partitions and topics, and several aspects of versions and configurations. This section discusses related work on each of these notions along with demonstrating the ways in which contexts can be applied to support these notions. Since authorization is a key issue in all applications of contexts, we start by discussing related work on authorization models.

Authorization. The authorization model adopted for contexts follows the policy of discretionary, content-based models. Users, arranged in a role hierarchy, own contexts and 
may provide access authorizations to other users, contexts and transactions, based on the contents of their contexts. Our model shares essential features--most importantly the content-based nature--with the model suggested in [Bertino94]. Both models allow to express authorizations in terms of authorization predicates. This is in contrast to models that define all authorizations in the form of a matrix that does not allow one to examine the contents of an object for the purpose of deriving authorization decisions.

Like other advanced discretionary models, such as those reported in [Rabitti91] or [Jonscher93], our model is based on hierarchies of user roles (authorization subjects) as well as authorization objects. Two distinguishing features of our model are the flexible grain-size of authorization objects and the reliance on transaction hierarchies rather than on hierarchies of (primitive) access types. Since contexts themselves form units of authorization and can be constructed to contain contexts, such as those that form the components of a composite object or others that are versions, any arbitrary grain-size of authorization objects is accommodated. Note that this is not the case in authorization models designed for object-oriented data models since these employ a predefined hierarchy of authorization objects.

Workspaces. In the field of software development, commercial tools like Apollo DSEE [Leblang84] or SUN NSE [SUN88] provide basic support for workspaces. These tools, however, support authorization only at a very coarse level and are constrained to deal with change notification. In the field of object-oriented development, Wile and Allard [Wile86] propose worlds as a mechanism to combine the advantages of conventional file systems, such as the data sharing mechanisms, with object-bases. The resulting structures, worlds, describe clusters of information in an object-base. In [Motschnig-Pitrik90], perspectives have been proposed as one basic structuring and abstraction mechanism within SFW, an abstract structuring framework. Perspectives in SFW, like in the framework presented here, allow to filter out relevant information on conceptual entities while discarding irrelevant details. A recent approach to partitioning in the context of software development environments is the concept of "perspectives" as introduced in [Prevelakis93]. "Perspectives" are embedded in an object management system and come equipped with similar, powerful, set-based construction operations as do contexts.

The approach presented here, however, differs with respect to the basic data structure (called lexicon) used to keep track of information units scattered across contexts and with respect to handling authorization. Also, we suggest a generic model of change propagation that generalizes models of view updates in databases and extends change propagation facilities of workspaces models [Katz90]. Besides sharing, authorization, and change propagation, a workspace model must provide means for information hiding [Katz90]. Using the context mechanism, information hiding is achieved in one of two ways. First, authorization predicates can be used to "hide" (portions of) contexts with respect to other contexts, users, or transactions. Alternatively, users may be forced to work in contexts that have been created by a difference or masking operation thus blending out units to be hidden. 
User Views. Database views constitute a well known technique of partitioning as well as integrating databases. Since the functionality of a view concept largely depends on the underlying data model, it is instructive to compare the modelling of views in the context framework with view mechanisms found in advanced data models such as those for object-oriented databases (OODBs) (see, for example [Scholl91], [A biteboul91], [Bertino92], [Rundensteiner92], [dos Santos94], and [Motschnig-Pitrik96] for a survey). Contexts share with object preserving approaches to OO views (for example, [Scholl 91], [Rundensteiner92]) the advantage of not having to cope with the view update problem ${ }^{5}$ [Gottlob88]. As discussed in [Scholl91], this is because objects in $O O$ notations have an identity independent of their associated values. Thus, if the view definition language preserves object identity, view updates can unambiguously be traced back to their parent objects. It is apparent that the object preservation property holds for the operations used to create and extend contexts. This follows immediately from the fact that these operations manipulate the contents and the lexicon of contexts without ever creating new referents. The latter can only be created using operations that an information provides for the purpose of introducing objects into the information base.

In fact, views constitute but one constellation of contexts that can be developed into a pattern for designing contexts. We first suggest a basic pattern and then show along which dimensions it can be extended. According to a basic pattern, an IB includes one global context that plays the role of the global IB. GlobalCtx includes any arbitrary number of (directly or indirectly) derived contexts. Authorization predicates are specified such that the global context and all those derived contexts that model updateable views authorize any transaction to be performed on them. Derived contexts modelling non updateable views, however, are assigned an authorization predicate that only allows queries to be performed on them, i.e. $\operatorname{author} P(u, t)=$ query $(t)$. Further, the owners of derived contexts modelling non updateable views must also be prevented from updating their contexts. Change propagation links are defined such that all changes from the global context are propagated to all derived contexts, but changes from contexts modelling updateable views are propagated only to the global context. An example of a global context called globalCtx and two view contexts, cv1 being updateable and cv2 not being updateable, is shown in figure 9 . Variations on the basic pattern may, for example, tighten access rights. In such a case contexts modelling views could be further constrained by the authorization mechanism such as to realize the concept of view-based authorization that is known from classical database systems.

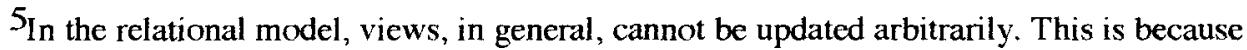
it is of ten ambiguous how to propagate view updates to updates on the base tuples. Thus, only views containing the key of their (single) base relation can be updated. 
IB

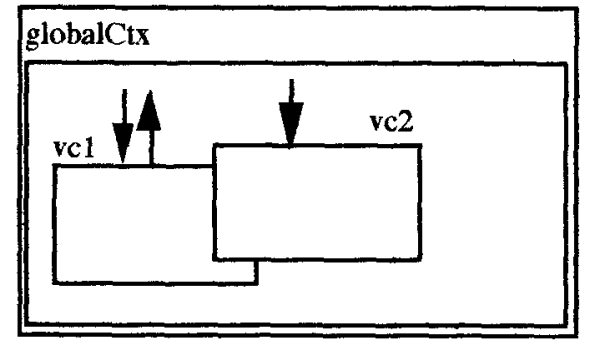

globalCtx:

propagationFrom(cProp,pred) $=\mathrm{vc} 1$ (cProp)

propagationTo(cProp,pred $)=\mathrm{cProp} \in\{\mathrm{vc1}, \mathrm{vc} 2)$

vc1:

propagationFrom(cProp,pred) $=$ globalCux(cProp)

propagation $\mathrm{T}$ (cProp,pred $)=$ globalCtx $(\mathrm{cProp})$

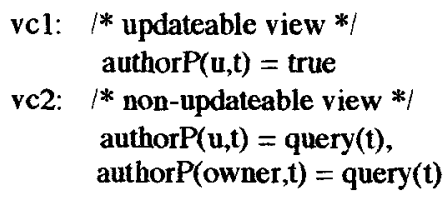

vc2:

propagationFrom (cProp,pred) $=$ globalCtx (cProp) propagationTo $(\mathrm{cProp}$, pred $)=$ false

Fig. 9: The use of contexts for modelling views in database systems as an example of a context-based design pattern.

Versions. The general way in which contexts serve to model versions is described in [Mylopoulos95]. In this place we would like to draw the reader's attention to the application of the particular embedding of the context mechanism into Telos. This is because advanced version modelling features tend to depend on the underlying information model [Katz90], [Ahmed91]. In order to illustrate this situation, below we show how two characteristic data model related issues can be mapped into Telos, extended to capture contexts. First recall that advanced approaches to version modelling consider the distinction of three categories of attributes:

* invariant attributes; these attributes may not be modified at the version level;

* Version-significant attributes; the update of a version-significant attribute causes a new version of the respective object to be created;

* nonversion-significant attributes may freely be updated without causing a new version to be generated.

The semantics of all three attribute categories can be captured within Telos by the mechanism of attribute metaclasses. For this reason, an attribute metaclass invariant is defined that postulates that no authorization predicate that allows for a modification may be defined on an invariant attribute. Any attribute within a particular context that shall be classified as invariant, is then made an instances of the "invariant" metaclass by associating it with the invariant attribute category. In a similar vein, the metaclass versionsignificant is introduced to require the creation of a new version (via the clone operation), once an attribute assigned the versionsignificant category is updated. Finally, assume that the nonversionsignificant category is the default since it does not impose any restrictions.

Second, consider the distinction of three version states in terms of the manipulations allowed in each of these states [Ahmed91]: 
* a version being in the validated state may not be updated; new versions may be derived from it;

* only nonversion-significant attributes of a version being in the stable state may be modified, new versions may be derived;

* all except invariant attributes may be modified in transient versions; new versions may not be derived.

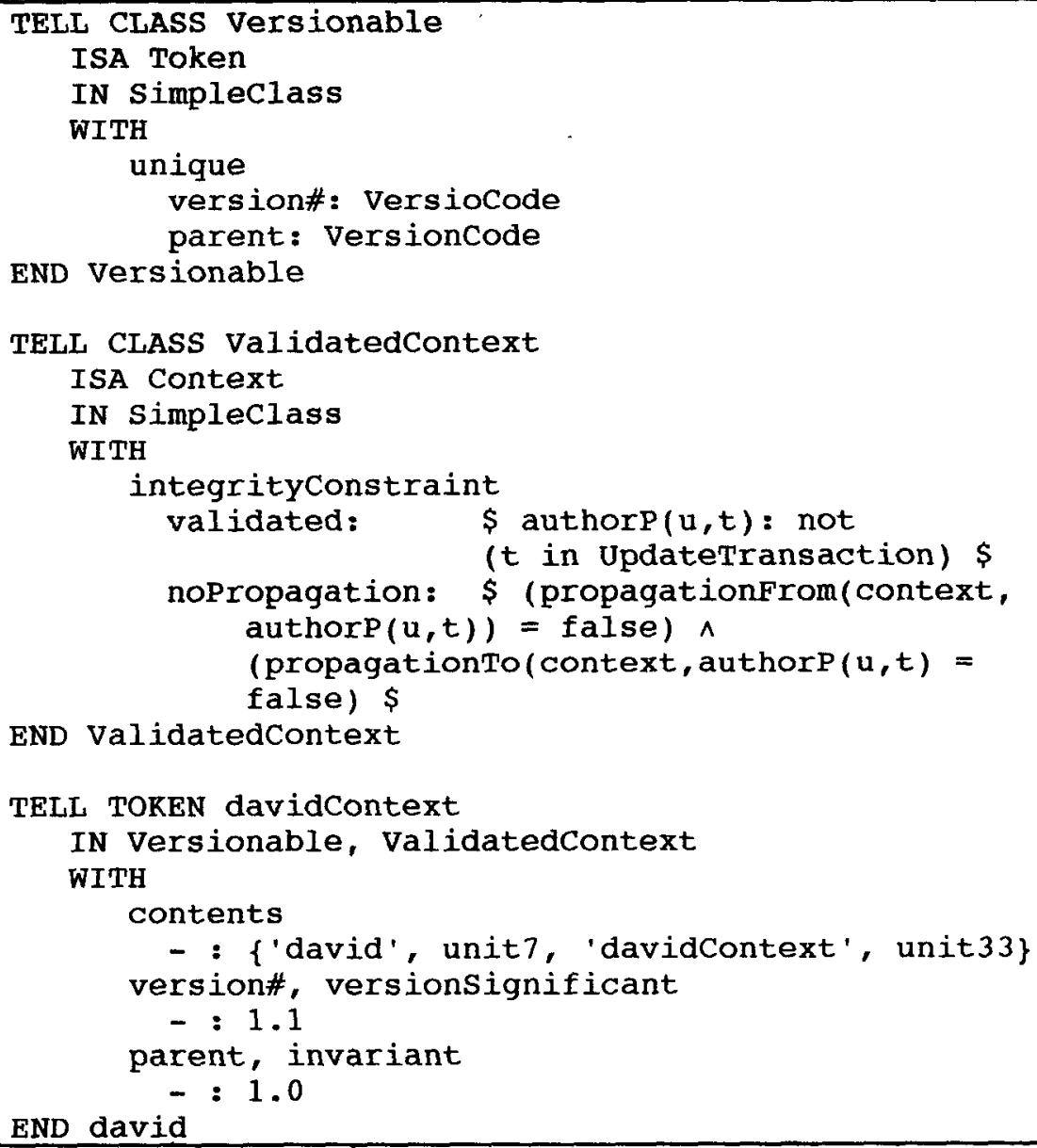

Fig. 10: Example showing the realization of versionable objects via contexts in Telos

In Telos, the following restrictions, respectively, can be imposed on contexts in order to model states corresponding to those described above:

* $\quad$ in a validated context, no modifying transactions are authorized; no change propagation channels may be defined;

* $\quad$ in a stable context, modifying transactions are authorized only on nonversionsignificant attributes; changes received via change propagation links may effect only nonversion-significant attributes; 
in a transient context, modifying transactions are not authorized on invariant attributes; transactions performing the "clone" operation are not authorized; changes received via change propagation links may not effect invariant attributes;

In order to demonstrate version modelling via contexts in Telos, figure 10 shows the class validatedcontext as a subclass of the Context. Further, the class versionable is introduced to allow for the instantiation of version objects with version numbers and links to their repective parents. Note that the class validatedcontext implements constraints on authorization and change propagation. On the bottom of figure 10 , the validated version of the context davidContext that encapsulates an instance of the class Person in form of a versionable context, is included in order to illustrate the interplay of the predefined classes used for the modelling of versions.

\section{Conctusions}

This paper suggests viewpoints as an abstraction mechanism which can be used to partition an information base into fragments, called contexts, according to varions criteria such as views, workspaces, versions and topics. This versatility of contexts is not surprising, since research in cognitive science has proved that viewing, essentially focusing on relevant information while discarding irrelevant issues, considerably aids understandability. These findings have been confirmed by linguistics. For linguists, the consideration of a context has for a long time been vital in order to allow one to derive the proper meaning of a word. The major contribution of this work has been to show that the viewpoint abstraction is amenable to sufficient formalization such as to be incorporated into current information models. In this respect it was shown how a generic framework for partitioning information bases into possibly overlapping contexts can be incorporated into the structurally object-oriented language Telos. In particular, the application of our framework to issues such as workspaces, database views, and features of version models has been discussed. It was shown in which ways these applications depend on the features designed into the context mechanism, such as operations for context construction, means to obtain relativity with respect to transaction executions, authorization and access control, encompassing the notion of privacy, and change propagation.

Furthermore, some applications of contexts were presented and illustrated by examples. In general, this work showed the feasibility of a unifying notion and accompanying support mechanisms for partitioning and focus of attention mechanisms which can be found in many areas of computer science. Further research will investigate guidelines for context construction that support the construction of pragmatically useful topologies of units that can be captured by contexts. Also, we are in the process of designing a metamodel for the viewpoint abstraction taking into account various factors that characterize viewpoints such as agents, biases, granularity levels, notations, and temporal aspects [Jarke96]. Furthermore, the adaptation of algorithms for efficient, incremental maintenance of contexts such as those proposed by [Dong92] and [Gupta93] will be addressed. We also intend to incorporate into Telos advanced, data-model dependent issues regarding version management, composite objects ([Kim89], [Ahmed91]), and authorization implication rules [Bertino94]. 


\section{References}

[Abiteboul91] Abiteboul, S., Bonner A.: "Objects and Views"; Proc. of A CM SIGMOD International Conference on the Management of Data, $238-247,1991$.

[Ahmed91] Ahmed R., Navathe S., B.: "Version Management of Composite Objects in CAD Databases"; Proc. ACM-SIGMOD Conference, 218-227, Denver, Colorado, May 1991.

[Anderson90] Anderson, J., Cognitive Psychology and its Implications, third edition, Freeman, 1990.

[Bertino92] Bertino E.: "A View Mechanism for Object-Oriented Databases"; EDBT 92'; Vienna, April 1992, Lecture Notes in Computer Science, 1992.

[Bertino94] Bertino E., Weigand H.. " An approach to authorization modeling in object-oriented database systems"; Data \& Knowledge Engineering, 12 (1), 1-29, February 1994.

[Brodie84] Brodie, M.: "On the Development of Data Models"; in: Brodie, M., Mylopoulos, J., Schmidt J., W., (eds.), On Conceptual Modeling, Springer, 1984.

[Dong92] Dong G., Topor R.: "Incremental Evaluation of Datalog Queries"; ICDT 92', 1992.

[dos Santos 94] dos Santos C. S., Abiteboul S., Delobel C.: "Virtual schemas and bases"; in Jarke, Bubenko, Jeffery (ed.), Advances in Database Technology - EDBT '94, 81-94, Springer, 1994.

[Gerst193] Gerstl P.: "Linking Linguistic and Non-Linguistic Information"; in R. P. van de Riet, R. A. Meersman, ed.: Linguistic Instruments in Knowledge Engineering", Elsevier Science Publishers, 1992.

[Gottlob88] Gottlob G., Paolini P., Zicari R.: "Properties and Update Semantics of Consistent Views"; ACM TODS, Vol.13, No.4, 486 - 521, December 1988.

[Gupta93] Gupta A., Mumick I., S., Subrahmanian V., S.: "Maintaining Views Incrementally"; SIGMOD 93', 1993.

[Hull87] Hull, R. and King, R., "Semantic Database Modelling: Survey, Applications and Research Issues", ACM Computing Surveys 19(3), September 1987.

[Jarke92] Jarke M., Mylopoulos J., .Schmidt J.W., Vassiliou Y.: "DAIDA: An Environment for Evolving Information Systems"; ACM Transactions on Information Systems, Vol.10, No.1, 1-15, January 1992.

[Jarke96] Jarke M., Gebhardt M., Nissen H. : personal communication.

[Jonscher93] Jonscher D., Dittrich K.,R.: "A Formal Security Model Based on an Object-Oriented Data Model"; Technical Repont No. 93.41, Institut fuer Informatik, Universitaet Zuerich, November 1993. 
[Katz90] Katz. R., H.: "Toward a Unified Framework for Version Modeling in Engineering Databases"; ACM Computing Surveys, Vol.22, No.4, 375-408, Dec. 1990.

[Kim89] Kim, W., Bertino, E., Garza, J., F., "Composite Objects Revisited"; OOPSLA 89', 337-347, 1989.

[Koubarakis89] Koubarakis, M., Mylopoulos, J., Stanley, M., Borgida, A., "TELOS: Features and Formalization", KRR-TR-89-4, Univ. of Toronto, Feb. 1989.

[Leblang84] Leblang D., B., Chase R., P.: "Computer-aided Software Engineering in a Distributed Workstation Environment", Proc. of the ACM SIGPLAN/SIGSOFT Conf. on Pract Software Development Environments, ACM, New York, 104-112, April 1984.

[Levesque84] Levesque, H., A Logic of Implicit and Explicit Belief, Proc. of the National Conference on Artificial Intelligence, Austin, Texas, pp. 198 - 202, Aug. 1984.

[Lewis76] Lewis, M., W., Anderson J., R., Interference with real world knowledge. Cognitive Psychology, 7, pp.311 - 335, 1976.

[Motschnig-Pitrik90] Motschnig-Pitrik R.: "A Framework for the Support of a Common Structural Level for Software, Database-, and Knowledge Based Systems"; The Journal of Systems and Software, North Holland, (12)12, 125-137, December 1990.

[Motschnig-Pitrik92] Motschnig-Pitrik R., Mylopoulos, J.: "Classes and Instances"; Int. Journal on Intelligent and Cooperative Information Systems, Vol.1, No. 1, 61-92, 1992.

[Motschnig-Pitrik93] Motschnig-Pitrik R.: "The Semantics of Parts Versus Aggregates in Data/Knowledge Modelling"; Proceedings of the CAiSE'93, Lecture Notes in Computer Science No. 685, 352-373, Springer, 1993.

[Motschnig-Pitrik95] Motschnig-Pitrik R.: "An Integrating View on the Viewing Abstraction: Contexts and Perspectives in Software Development, AI, and Databases";.Journal of Systems Integration, Kluwer, 5(1), 23-60, April 1995.

[Motschnig-Pitrik96] Motschnig-Pitrik R.: "Requirements and Analysis of ViewMechanisms for Object-Oriented Databases", Information Systems, to appear, 1996.

[Mylopoulos80] Mylopoulos J., Bernstein P., Wong H., "A Language Facility for Designing Interactive, Database-Intensive Applications"; ACM Transactions on Database Systems, 5(2), June 1980.

[Mylopoulos90] Mylopoulos, J., Borgida, A., Jarke, M. and Koubarakis, M., "Telos: Representing Knowledge About Information Systems", ACM Transactions on Information Systems, Vol.8, No.4, 325-362, Oct. 1990.

[Mylopoulos95] Mylopoulos J., Motschnig-Pitrik R.: "Partitioning Information Bases with Contexts"; Proc. of the 3rd International Conference on Cooperative Information Systems, 44-54, Vienna, May 1995 
[Prevelakis93] Prevelakis V., Tsichritzis D.: "Perspectives on Sof tware Development Environments"; Rolland C. et al. (editors), Proc. of the CAiSE '93, Paris, France, June 1993, Lecture Notes in Computer Science 685, Springer Verlag, 1993.

[Rabitti91] Rabitti F., Bertino E., Kim W., Woelk D.: " A Model of Authorization for Next-Generation Database Systems", ACM TODS, 16 (1), 88-131, March 1991.

[Rothkopf66] Rothkopf E., Z., Learning from Written Instruction Materials: An Explanation of the Control of Inspection Behavior by Test-like Events, American Educational Research Journal, 3, pp.241 - 249, 1966.

[Rundensteiner92] Rundensteiner E.: "MultiView: A Methodology for Supporting Multiple Views in Object-Oriented Databases"; Proc. of the 18th International Conference on Very Large Databases (VLDB), Vancouver, 1992.

[Schol191] Scholl M., H., Laasch C., Tresch M.: "Updateable Views in Object Oriented Databases"; Proc. of the 2nd Conf on DOOD, Munich, Dec. 1991.

[Schneider80] Schneider, P., F., Contexts in PSN, Proc. of the AI-CSCSI-SCEIO Conference, pp.71-78, Victoria, B.C., May 1980.

[Shaw84] Shaw M:: "The Impact of Modelling and Abstraction Concerns on Modern Programming Languages"; in: Brodie, M., Mylopoulos, J., Schmidt J., W., (ed.), On Conceptual Modeling, Springer Verlag, 1984.

[Smith77] Smith J.M., Smith D.C.P.: "Database Abstractions: Aggregation and Generalization"; ACM Trans. on Database Systems, Vol.2, No.2, 105-133, June 1977.

[Storey93] Storey V., C.: "Understanding Semantic Relationships"; inVery Large Data Bases Journal, Vol.2, No.4, 455-488, October 1993.

[SUN88] SUN Microsystems. "Introduction to the NSE.SUN Part No. 800-23621300, March 1988.

[Wand91] Wand Y., Weber R.: "A Unified Model of Software and Data Decomposition", Proc. of the 12th Annual International Conference on Information Systems, 101-110, New York, December 1991.

[Wile86] Wile, D., S., Allard, D., G., Worlds: an Organizing Structure for ObjectBases, ACM SIGPLAN Notices, Vol.22, No.1, 16-26, Jan. 1986.

[Yordon79] Yourdon E. and Constantine L.L.: "Structured Design - Fundamentals of a Discipline of Computer Program and Systems Design", Prentice Hall, 1979. 